\title{
On the existence of a gyroscope in spaces with affine connections and metrics
}

\author{
S. Manoff*, B. Dimitrov \\ Joint Institute for Nuclear Research, \\ Bogoliubov Laboratory of Theoretical Physics, \\ Dubna, Moscow Region \\ 141980 Russia \\ (e-mail addresses: smanov@thsun1.jinr.ru,smanov@inrne.bas.bg,bogdan@thsun1.jinr.ru)
}

\begin{abstract}
Conditions for the existence of a gyroscope in spaces with affine connections and metrics are found. They appear as special types of Fermi-Walker transports for vector fields, lying in a subspace, orthogonal to the velocity vector field (a non-null contravariant vector field) of an observer.

PACS numbers: 04.20Cv, 04.90.+e, 04.50.+h, 02.40.Ky
\end{abstract}

\section{INTRODUCTION}

In the last years spaces with affine connections and metrics [1] $\div$ [3] have deserved some interest related to the possibility of using mathematical models of space-time different from (pseudo) Riemannian spaces. The main reasons for new models of the space-time are generated mostly by [4]:

(a) attempts to quantize gravity,

\footnotetext{
*Permanent address: Bulgarian Academy of Sciences, Instutute for Nuclear Research and Nuclear Energy, Department of Theoretical Physics, Blvd. Tzarigradsko Chaussee 72, 1784 Sofia - Bulgaria
} 
(b) attempts for description of "hadron (or nuclear) matter in terms of extended structure" [4], [5],

(c) attempts for modelling the early universe,

(d) models of continuous media with microstructure,

(e) models of classical and quantum field theories with more comprehensive structure of the corresponding space-time.

The use of spaces with affine connections and metrics has been critically evaluated from different points of view [6], [7]. There are at least four major objections against the applications of this types of spaces in physics:

1. The violation of the equivalence principle related to the non-possibility of bringing to zero the components of an affine connection on a curve in the space-time,

2. The non-preservation (deformation) of a Lorentz basis along a geodesics as a result of the non-compatibility of the affine connections (the parallel transports) with the metrics (the measurement of lengths). This means that in space with affine connections and metrics there are no transports along a vector field preserving the lengths of vector fields and angles between vector fields, transported along it,

3. The deformation of a light cone leading to the abuse of the law of causality considered as a basic law in classical physics,

4. The independence of the affine connections and the metrics from each other could lead to the determination of the affine connections in twofold manner: on the one side, through the solution of some conditions for compatibility [s. p. 2.] and, on the other side, through Lagrangian formalism for both type of dynamic variables (the components of the affine connections and the components of the metrics).

In the last few years the first three objections $(1 . \div 3$. $)$ have been removed by the investigations of different authors.

1a. It has been proved that in spaces with affine connections (whose components differ only by sign or not only by sign) and metrics [i.e. in the so called $\left(L_{n}, g\right)$ - and $\left(\bar{L}_{n}, g\right)$-spaces] the principle of equivalence holds 8$] \div[12]$, [13]. 
2a. In spaces with affine connections and metrics special types of transports (called Fermi-Walker transports) [14 $\div$ [16 exist which do not deform a Lorentz basis,

3c. There also exist other type of transports (called conformal transports) [17], [18] under which a light cone does not deform.

4a. The auto-parallel equation can play the same role in spaces with affine connections and metrics for describing the motion of a free spinless test particles as the geodesic equation does in Einstein's theory of gravitation [19], 20].

\section{A. Problems and results}

The main purpose of the present paper is to show that the last objection 4. could also be removed by means of the proof of the existence of a gyroscope in spaces with affine connections and metrics. A gyroscope is characterized by its three axes (in a 3- or 4dimensional space-time) and the angles between them. The length of the axes and the angles between them should not change, when the gyroscope moves in the time or in the space-time. In this sense a gyroscope represents a rigid body, determined by its axes [21], [22]. The existence of a gyroscope is related to the fact that special types of Fermi-Walker transports could be found, which exist for every preliminary given contravariant non-null vector field with its corresponding projective metrics. For these types of transports the length of the $n-1(n=4)$ gyroscope's axes lying in the $n-1$ dimensional subspace could move in the time without changing their lengths and the angles between them. The non-null contravariant vector field could be interpreted as the velocity of an observer and the vectors, orthogonal to it as the axes of a gyroscope. In this case, the independent to each other affine connections and metrics would fulfill automatically compatibility conditions for the special type of transports (related to the affine connections) and the measurements of length (related to the metrics). The affine connections and the metrics could be found uniquely by the use of a Lagrangian formalism or by other methods common in physics.

Let us now consider the change of the length of a vector along a non-null contravariant 
vector field in a space with affine connections and metrics. The measuring of the length and its changes in space-time is very important for theory and experiment in physics.

\section{FERMI-WALKER TRANSPORTS (FWT) IN SUBSPACES WITH PROJECTIVE METRICS}

Let a contravariant affine connection $\Gamma$ and a covariant affine connection $P$ be given [3] with components in a co-ordinate basis given respectively as $\Gamma_{j k}^{i}$ and $P_{j k}^{i}$ over a differentiable manifold $M$ with $\operatorname{dim} M=n$. For $\left(L_{n}, g\right)$-spaces $P_{j k}^{i}=-\Gamma_{j k}^{i}$. For $\left(\bar{L}_{n}, g\right)$-spaces $P_{j k}^{i}+\Gamma_{j k}^{i}=$ $g_{j ; k}^{i} \neq 0$, where $g_{j}^{i}$ are the components of the Kronecker tensor $K r=g_{j}^{i} \cdot \partial_{i} \otimes d x^{j}$. Let $M$ be provided with a covariant metric $g=g_{i j} \cdot d x^{i} \cdot d x^{j}, g_{i j}=g_{j i}, d x^{i} \cdot d x^{j}=(1 / 2)$. $\left(d x^{i} \otimes d x^{j}+d x^{j} \otimes d x^{i}\right)$ and its corresponding contravariant metric $\bar{g}=g^{i j} \cdot \partial_{i} . \partial_{j}, g^{i j}=g^{j i}$, $\partial_{i} . \partial_{j}=(1 / 2) \cdot\left(\partial_{i} \otimes \partial_{j}+\partial_{j} \otimes \partial_{i}\right)$. Let a non-null (non-isotropic) vector field $u$ be given with $g(u, u)=e= \pm l_{u}^{2} \neq 0$, where $\bar{g}(g)(u)=\bar{g}[g(u)]=u$. The change of the length $l_{\xi}=|g(\xi, \xi)|^{1 / 2}$ of another contravariant non-null vector field $\xi$ along the vector field $u$ could be found in the form [23]

$$
u l_{\xi}= \pm \frac{1}{2 \cdot l_{\xi}} \cdot\left[\left(\nabla_{u} g\right)(\xi, \xi)+2 \cdot g\left(\nabla_{u} \xi, \xi\right)\right], \quad l_{\xi}: \neq 0
$$

where $u l_{\xi}=u^{i} \cdot \partial_{i} l_{\xi}=u^{i} \cdot\left(\partial / \partial x^{i}\right) l_{\xi}, \nabla_{u} \xi$ is the covariant derivative of $\xi$ along $u$, and $\nabla_{u} g$

is the covariant derivative of $g$ along $u$. Both covariant derivatives are with respect to the affine connections $\Gamma$ and $P$.

The change of the angle (the cosine of the angle respectively) between two contravariant non-null vector fields $\xi$ and $\eta$ along the vector field $u$ could be written in the form [23]

$$
\begin{aligned}
u[\cos (\xi, \eta)]= & \frac{1}{l_{\xi} \cdot l_{\eta}} \cdot\left[\left(\nabla_{u} g\right)(\xi, \eta)+g\left(\nabla_{u} \xi, \eta\right)+g\left(\xi, \nabla_{u} \eta\right)\right]- \\
& -\left[\frac{1}{l_{\xi}} \cdot\left(u l_{\xi}\right)+\frac{1}{l_{\eta}} \cdot\left(u l_{\eta}\right)\right] \cdot \cos (\xi, \eta) .
\end{aligned}
$$

The conditions for transports of the covariant metric $g$ and the conditions for transports

of the contravariant vector fields $\xi$ and $\eta$ as well determine the change of the lengths of the two vector fields as well as the angle between them. 
To the vector field $u$ correspond its covariant projective metric $h_{u}=g-(1 / e) \cdot g(u) \otimes g(u)$ and its contravariant projective metric $h^{u}=\bar{g}-(1 / e) \cdot u \otimes u$.

If a vector field $\xi$ is orthogonal to the vector field $u$, i.e. if $g(u, \xi):=0$, then $\xi$ could be written as $\xi_{\perp}=\bar{g}\left[h_{u}(\xi)\right]=g^{i j} \cdot h_{\overline{j k}} \cdot \xi^{k} \cdot \partial_{i}$ in a $\left(\bar{L}_{n}, g\right)$-space. The change $u l_{\xi_{\perp}}$ of the length $l_{\xi_{\perp}}$ of $\xi_{\perp}$ could be found as

$$
u l_{\xi_{\perp}}= \pm \frac{1}{2 \cdot l_{\xi_{\perp}}} \cdot\left[\left(\nabla_{u} h_{u}\right)\left(\xi_{\perp}, \xi_{\perp}\right)+2 \cdot h_{u}\left(\nabla_{u} \xi_{\perp}, \xi_{\perp}\right)\right]
$$

where

$$
l_{\xi_{\perp}}^{2}= \pm h_{u}\left(\xi_{\perp}, \xi_{\perp}\right), \quad \quad l_{\xi_{\perp}}=\left|h_{u}\left(\xi_{\perp}, \xi_{\perp}\right)\right|^{1 / 2}
$$

The change of the angle between two orthogonal to $u$ vector fields $\xi_{\perp}\left[\right.$ with $g\left(u, \xi_{\perp}\right)=0$ ] and $\eta_{\perp}\left[\right.$ with $\left.g\left(u, \eta_{\perp}\right)=0\right]$ could be computed and presented in the form

$$
\begin{aligned}
u\left[\cos \left(\xi_{\perp}, \eta_{\perp}\right)\right]= & \frac{1}{l_{\xi_{\perp}} \cdot l_{\eta_{\perp}}} \cdot\left[\left(\nabla_{u} h_{u}\right)\left(\xi_{\perp}, \eta_{\perp}\right)+h_{u}\left(\nabla_{u} \xi_{\perp}, \eta_{\perp}\right)+h_{u}\left(\xi_{\perp}, \nabla_{u} \eta_{\perp}\right)\right]- \\
& -\left[\frac{1}{l_{\xi_{\perp}}} \cdot\left(u l_{\xi_{\perp}}\right)+\frac{1}{l_{\eta_{\perp}}} \cdot\left(u l_{\eta_{\perp}}\right)\right] \cdot \cos \left(\xi_{\perp}, \eta_{\perp}\right) .
\end{aligned}
$$

The expressions for $u l_{\xi_{\perp}}$ and $u\left[\cos \left(\xi_{\perp}, \eta_{\perp}\right)\right]$ contain only the covariant projective metric $h_{u}$, its covariant derivative along $u$ and the corresponding vector fields $\xi_{\perp}$ and $\eta_{\perp}$ as well as their covariant derivatives $\nabla_{u} \xi_{\perp}, \nabla_{u} \eta_{\perp}$, and the derivatives $u l_{\xi_{\perp}}$ and $u l_{\eta_{\perp}}$ along $u$.

The question arises under which conditions for $\nabla_{u} h_{u}, \nabla_{u} \xi_{\perp}$, and $\nabla_{u} \eta_{\perp}$ the relations

$$
u l_{\xi_{\perp}}=0, \quad u l_{\xi_{\perp}}=0, \quad u\left[\cos \left(\xi_{\perp}, \eta_{\perp}\right)\right]=0
$$

are valid, i.e. under which conditions the lengths of the vector fields $\xi_{\perp}$ and $\eta_{\perp}$, as well as the angle between them, do not change under a transport along the vector field $u$. Transports which preserve lengths and angles between vector fields are called Fermi-Walker transports [14], [17]. We can now apply the method, developed for finding out Fermi-Walker transports in spaces with affine connections and metrics with given metrics $g$ and $\bar{g}$, to the same type of spaces with determined projective metrics $h_{u}$ and $h^{u}$. This method [in details 
described in [16]] is related to the introduction of an extended covariant differential operator ${ }^{e} \nabla_{u}=\nabla_{u}-\bar{A}_{u}$. The quantity $\bar{A}_{u}$ is a tensor of the type

$$
\bar{A}_{u}=\bar{g}(C(u))=g^{i k} \cdot C_{\bar{k} j}(u) \cdot \partial_{i} \otimes d x^{j}
$$

As a mixed tensor of second rank (depending on $u$ ) $\bar{A}_{u}$ has to obey certain conditions under which if ${ }^{e} \nabla_{u} \xi_{\perp}=\nabla_{u} \xi_{\perp}-\bar{A}_{u} \xi_{\perp}=0$, i.e. if $\nabla_{u} \xi_{\perp}=\bar{A}_{u} \xi_{\perp}=\bar{g}(C(u))\left(\xi_{\perp}\right)$, then $u l_{\xi_{\perp}}=0, \quad u l_{\xi_{\perp}}=0$, and $u\left[\cos \left(\xi_{\perp}, \eta_{\perp}\right)\right]=0$.

Using the relation $\bar{g}=h^{u}+(1 / e) \cdot u \otimes u$, we can represent $\nabla_{u} \xi_{\perp}=\bar{A}_{u} \xi_{\perp}$ in the form

$$
\nabla_{u} \xi_{\perp}=h^{u}(C(u))\left(\xi_{\perp}\right)+\frac{1}{e} \cdot[(u)(C(u))]\left(\xi_{\perp}\right) \cdot u
$$

After introducing the last expression in the relation for $u l_{\xi_{\perp}}$ and after some calculations we can find the relations

$$
\begin{gathered}
u l_{\xi_{\perp}}= \pm \frac{1}{2 \cdot l_{\xi_{\perp}}} \cdot\left\{\left(\nabla_{u} h_{u}\right)\left(\xi_{\perp}, \xi_{\perp}\right)+2 \cdot h_{u}\left[h^{u}(C(u))\left(\xi_{\perp}\right), \xi_{\perp}\right]\right\} \\
{\left[h_{u}\left(h^{u}\right)(C(u))\right]_{s}=-\frac{1}{2} \cdot \nabla_{u} h_{u}}
\end{gathered}
$$

where

$$
\begin{aligned}
h_{u}\left(h^{u}\right)(C(u)) & =h_{i k} \cdot h^{\overline{k l}} \cdot C_{k j}(u) \cdot d x^{i} \otimes d x^{j}= \\
& =h^{\overline{k l}} \cdot h_{i k} \cdot C_{k j}(u) \cdot d x^{i} \otimes d x^{j}=h^{u}\left[h_{u} \otimes C(u)\right], \\
h_{u}\left[h^{u}(C(u))\left(\xi_{\perp}\right), \xi_{\perp}\right] & =\left\{h^{u}\left[h_{u} \otimes C(u)\right]\right\}\left(\xi_{\perp}, \xi_{\perp}\right), \\
{\left[h_{u}\left(h^{u}\right)(C(u))\right]_{s} } & \left.=\frac{1}{2} \cdot\left\{h^{u}\left[h_{u} \otimes C(u)\right]+h^{u}\left[C(u) \otimes h_{u}\right)\right]\right\}, \\
{\left[h_{u}\left(h^{u}\right)(C(u))\right]_{a} } & \left.=\frac{1}{2} \cdot\left\{h^{u}\left[h_{u} \otimes C(u)\right]-h^{u}\left[C(u) \otimes h_{u}\right)\right]\right\} .
\end{aligned}
$$

If (9) is fulfilled, then $u l_{\xi_{\perp}}=0$. Therefore, to every Fermi-Walker transport with a given tensor $C(u)$ there exists a corresponding Fermi-Walker transport for the orthogonal to the vector field $u$ contravariant vector fields $\left\{\xi_{\perp} \in T^{\perp u}(M)\right\}$. On the other side, to every given tensor $C(u)$, i.e. to every given extended covariant differential operator ${ }^{e} \nabla_{u}=\nabla_{u}-\bar{g}(C(u))$, 
there exists a Fermi-Walker transport for the orthogonal to $u$ contravariant vector fields $\left\{\xi_{\perp} \in T^{\perp u}(M)\right\}$. A Fermi-Walker transport of this type is described by the condition (9) or in a co-ordinate basis by the condition

$$
h_{i k} \cdot h^{\overline{k l}} \cdot C_{l j}(u)+h_{j k} \cdot h^{\overline{k l}} \cdot C_{l i}(u)=-h_{i j ; k} \cdot u^{k}
$$

Since [16] $C_{l j}(u)=\left(A_{l j \bar{k}}+B_{l j ; k}\right) \cdot u^{k}$, we have for every arbitrary given non-null vector field $u$ the conditions for $h_{i j}$

$$
h_{i j ; k}=-\left[h_{i k} \cdot h^{\overline{k l}} \cdot\left(A_{l j \bar{k}}+B_{l j ; k}\right)+h_{j k} \cdot h^{\overline{k l}} \cdot\left(A_{l i \bar{k}}+B_{l i ; k}\right)\right] \text {, }
$$

where $A_{l j \bar{k}}$ and $B_{l j ; k}$ are the components of tensor fields determining the different types of Fermi-Walker transports in spaces with affine connections and metrics for given metrics $g$ and $\bar{g}$.

Taking into account the preservation of the lengths $l_{\xi_{\perp}}$ and $l_{\eta_{\perp}}$ under the above conditions for a FWT we can find the remaining conditions for the preservation of the angle between $l_{\xi_{\perp}}$ and $l_{\eta_{\perp}}$ under their transport along the vector field $u$. For $u\left[\cos \left(\xi_{\perp}, \eta_{\perp}\right)\right]$ we obtain [if $\left.u l_{\xi_{\perp}}=0, u l_{\xi_{\perp}}=0\right]$

$$
\begin{aligned}
u\left[\cos \left(\xi_{\perp}, \eta_{\perp}\right)\right]= & \frac{1}{l_{\xi_{\perp}} \cdot l_{\eta_{\perp}}} \cdot\left\{\left(\nabla_{u} h_{u}\right)\left(\xi_{\perp}, \eta_{\perp}\right)+h_{u}\left[h^{u}(C(u))\left(\xi_{\perp}\right), \eta_{\perp}\right]+\right. \\
& \left.+h_{u}\left[h^{u}(C(u))\left(\eta_{\perp}\right), \xi_{\perp}\right]\right\} .
\end{aligned}
$$

From the relations

$$
\begin{aligned}
\left(\nabla_{u} h_{u}\right)\left(\xi_{\perp}, \eta_{\perp}\right) & =-2 \cdot\left[h_{u}\left(h^{u}\right)(C(u))\right]_{s}\left(\xi_{\perp}, \eta_{\perp}\right), \\
h_{u}\left[h^{u}(C(u))\left(\xi_{\perp}\right), \eta_{\perp}\right] & =\left[h_{u}\left(h^{u}\right)(C(u))\right]\left(\eta_{\perp}, \xi_{\perp}\right), \\
h_{u}\left[h^{u}(C(u))\left(\eta_{\perp}\right), \xi_{\perp}\right] & =\left[h_{u}\left(h^{u}\right)(C(u))\right]\left(\xi_{\perp}, \eta_{\perp}\right),
\end{aligned}
$$

after representing the last two expression in their symmetric and antisymmetric parts, it follows that $u\left[\cos \left(\xi_{\perp}, \eta_{\perp}\right)\right]=0$ is automatically fulfilled.

Therefore, in spaces with affine connections and metrics a gyroscope could exist if its axes $\xi_{\perp}^{(b)}[b=1, \ldots, n-1 ; n=3,4, \ldots]$ are transported under a Fermi-Walker transport along 
a worldline with a tangent vector $u$. The FWT for the axes of the gyroscope is determined by the condition

$$
\begin{aligned}
h_{u}\left(\nabla_{u} \xi_{\perp}^{(b)}\right) & =-\frac{1}{2} \cdot\left(\nabla_{u} h_{u}\right)\left(\xi_{\perp}^{(b)}\right)+\left[h_{u}\left(h^{u}\right)(C(u))\right]_{a}\left(\xi_{\perp}^{(b)}\right)= \\
& =-\frac{1}{2} \cdot\left(\nabla_{u} h_{u}\right)\left(\xi_{\perp}^{(b)}\right)+\left\{h^{u}\left[h_{u} \otimes C(u)\right]\right\}_{a}\left(\xi_{\perp}^{(b)}\right) .
\end{aligned}
$$

If we chose $\xi_{\perp}^{(b)}$ in a way that $£_{u} \xi_{\perp}^{(b)}=0$ (i.e. if $u$ and $\xi_{\perp}^{(b)}$ are tangent vectors to the co-ordinates in the space-time $M$ ), then [24], 25]

$$
\begin{aligned}
h_{u}\left(\nabla_{u} \xi_{\perp}^{(b)}\right) & =\left(\sigma+\frac{1}{n-1} \cdot \theta \cdot h_{u}\right)\left(\xi_{\perp}^{(b)}\right)+\omega\left(\xi_{\perp}^{(b)}\right)= \\
& =\left[h_{u}\left(h^{u}\right)(C(u))\right]_{s}\left(\xi_{\perp}^{(b)}\right)+\left[h_{u}\left(h^{u}\right)(C(u))\right]_{a}\left(\xi_{\perp}^{(b)}\right),
\end{aligned}
$$

where [24] the tensor $\sigma$ is the shear velocity tensor (shear), the invariant $\theta$ is the expansion velocity invariant (expansion), and $\omega$ is the rotation velocity tensor (rotation). From the last two expressions (for every arbitrary given vector $\xi_{\perp}$ on which the covariant differential operator $h_{u} \circ \nabla_{u}$ acts) we obtain the relations

$$
\begin{aligned}
& {\left[h_{u}\left(h^{u}\right)(C(u))\right]_{s}=\sigma+\frac{1}{n-1} \cdot \theta \cdot h_{u},} \\
& {\left[h_{u}\left(h^{u}\right)(C(u))\right]_{a}=\omega .}
\end{aligned}
$$

For a Fermi-Walker transport for $\xi_{\perp}$ along $u$, we have the condition in the form

$$
\nabla_{u} h_{u}=-2 \cdot\left(\sigma+\frac{1}{n-1} \cdot \theta \cdot h_{u}\right)
$$

\section{A. Fermi-Walker transports along a shear-free and / or expansion-free vector field $u$}

For a shear-free vector field $u(\sigma=0)$ the condition (18) for a FWT degenerates in the recurrent relation for $h_{u}$

$$
\nabla_{u} h_{u}=-\frac{2}{n-1} \cdot \theta \cdot h_{u}, \quad £_{u} \xi_{\perp}=0
$$

For a shear-free and expansion-free vector field $u(\sigma=0, \theta=0)$ [20] the condition (18) for a FWT degenerates in the condition for a parallel transport of $h_{u}$ along $u$ 


$$
\nabla_{u} h_{u}=0
$$

In this type of spaces with affine connections and metrics the parallel transport of two contravariant non-null vector fields $\xi_{\perp}$ and $\eta_{\perp}$ along the vector field $u$ assure the preservation of its lengths and angles between them.

\section{B. Some remarks}

1. If a (pseudo) Riemannian space (as a special case of a space with affine connections and metrics) admits a Killing vector field $u$, i.e. if $£_{u} g=0$, leading to $\sigma=\theta=0$, then a FWT is determined by the parallel transport of $h_{u}$.

2. The conditions for the existing of a gyroscope in spaces with affine connections and metrics do not determine the affine connections and the metrics. For every space with given affine connections and metrics there exist Fermi-Walker transports preserving the axes of a gyroscope and the angles between them. The conditions for a special FWT determine only the transport of the vector fields $\xi_{\perp}$, and $\eta_{\perp}$ along a given vector field $u$ in a given space-time. The compatibility between metrics (measurements of lengths) and affine connections (transports which are not geodesic) is automatically fulfilled by the choice of the corresponding transport preserving lengths and angles. Thus, the last objections for using spaces with affine connections and metrics as models of space-time is removed.

\section{CONCLUSIONS}

In the present paper special types of Fermi-Walker transports are consider under which a gyroscope can exist in spaces with affine connections and metrics. These types of transports are in general different from the geodesic transports but they play the same role as the geodesic transports in (pseudo) Riemannian spaces and, at the same time, do not put any conditions on the affine connections and metrics. The last two geometric objects could be determined by other methods uniquely since the compatibility conditions between them are 
fulfilled automatically under the special types of Fermi-Walker transports. Physical theories (including theories of gravitation) could be constructed in the above mentioned types of spaces not only for microphysics (quantum physics) but also for macrophysics (classical physics). 


\section{REFERENCES}

[1] A. P. Norden, Spaces with affine connection 2-nd ed. (Nauka, Moscow, 1976) (in Rus$\operatorname{sian})$.

[2] P. K. Raschewski, Riemannsche Geometrie und Tensoranalysis (VEB Deutscher Verlag d. Wissenschaften, Berlin, 1959).

[3] S. Manoff, Physics of Particles and Nuclei [Russian Edition: 30 5, 1211-1269 (1999)], [English Edition: 30 5, 527-549 (1999)].

[4] F. W. Hehl, J. D. McCrea, E. W. Mielke, Y. Ne'eman, Physics Reports 258 1-2, 1-171 (1995).

[5] R. D. Hecht, F W Hehl, Proc. 9th Italian Conf. on Gen. Rel. and Grav. Physics. Capri, Italy 1991. eds. Cianci R. et al. (World Sci. Pub. Co., Singapore, 1991), pp. 246-291.

[6] K. Hayashi, Phys. Lett. 65 B 5, 437-440 (1976).

[7] H.-H. von Borzeszkowski, H.-J. Treder, Gen. Rel. and Grav. 29 4, 455-466 (1997).

[8] B. Z. Iliev, J. Phys. A: Math. Gen. 29 6895-6901 (1996).

[9] B. Z. Iliev, J. Phys. A: Math. Gen. 30 4327-4336 (1997).

[10] B. Z. Iliev, J. Phys. A: Math. Gen. 31 1287-1296 (1998).

[11] B. Z. Iliev, Journal of Geometry and Physics 24 209-222 (1998).

[12] D. Hartley, Class. and Quantum Grav. 12 L103-L105 (1995).

[13] S. Manoff, Intern. J. Mod. Phys. A 11 21, 3849-3874 (1996).

[14] S. Manoff, Class. Quantum Grav. 15 2, 465-477 (1998).

[15] S. Manoff, JINR Rapid Communications 1 [81] 5-12 (1997).

[16] S. Manoff, Intern. J. Mod. Phys. A 13 25, 4289-4308 (1998). 
[17] S. Manoff, Intern. J. Mod. Phys. A 15 5, 679-695 (2000).

[18] S. Manoff, Intern. J. Math. Phys., Nonlinear Phenomena, and Group Theory (to appear), gr-qc/99 07095.

[19] S. Manoff, 2000 Auto-parallel equation as Euler-Lagrange's equation over spaces with affine connections and metrics Gen. Rel. and Grav. 32 8,1559-1582. E-print grqc/0010048

[20] S. Manoff, gr-qc/0011045.

[21] J. L. Synge, Relativity: the general theory (North-Holland Publ. Co., Amsterdam, 1960).

[22] J. Ehlers, Abhandlungen d. Mainzer Akademie d. Wissenschaften, Math.-Naturwiss. Kl. Nr. 11 (1961).(Engl. transl. Gen. Rel. and Grav. 25 12, 1225-1266 (1993).

[23] S. Manoff, gr-qc/0002073.

[24] S. Manoff, Complex Structures and Vector Fields. Eds. Dimiev St., Sekigawa K. (World Sci. Publ., Singapore, 1995), pp. 61-113.

[25] S. Manoff, gr-qc/0005027. 